\title{
Dielectric properties of human diabetic blood: Thermodynamic characterization and new prospective for alternative diagnostic techniques
}

\author{
F. Farsaci*, S. Ficarra ${ }^{\dagger}$, A. Russo ${ }^{\dagger}$, A. Galtieri ${ }^{\dagger}$ and E. Tellone ${ }^{\dagger, *}$ \\ *IPCF-C.N.R., Via Ferdinando Stagno d'Alcontres 37 \\ 98158 Faro Superiore, Messina, Italy \\ and \\ †Department of Chemical Sciences \\ University of Messina, Viale Ferdinando Stagno \\ d'Alcontres 31, 98166 Messina, Italy \\ tetellone@unime.it
}

Received 12 March 2015; Revised 9 April 2015; Accepted 9 June 2015; Published 2 July 2015

\begin{abstract}
In this paper, we will show the possibility of studying physical properties and irreversible phenomena that occur in blood by applying the dielectric Kluitenberg's nonequilibrium thermodynamic theory. Namely, we shall use some recent extensions of this theory that allow to infer its main characteristic parameters from experimental measures. Applying these results to the study of normal and diabetic blood we show, by comparing them, that it is possible to determine the difference, in some details, of the amount of particular phenomena occurring inside them and give a biological meaning to these phenomena. Moreover, observing a correspondence between a particular value of the frequency for which state coefficients are equal and glucose levels we introduce an alternative diagnostic method to measure the values of the glucose in the blood by determining only this frequency value. The thermodynamic description will be completed by determining the trend of the entropy production.
\end{abstract}

Keywords: Blood; dielectric relaxation in blood; nonequilibrium thermodynamics.

\section{Introduction}

Diabetes mellitus (DM) is a group of complex multisystem metabolic disorders characterized by a relative or absolute insufficiency of insulin secretion and/or concomitant resistance to the metabolic action of insulin on target tissues. Diabetes is an increasingly significant health problem as the prevalence increases with age, the lifetime risk of developing diabetes is around 10\%. ${ }^{1}$ According to the 1999 World Health Organization (WHO) criteria (which are also endorsed by Diabetes UK), diagnosis of diabetes is made if the fasting blood glucose level is $7.0 \mathrm{mmol} / \mathrm{L}$ or more, or a random blood glucose test shows a level of $11.1 \mathrm{mmol} / \mathrm{L}$ or more. People with diabetes have an increased risk of developing a number of serious health problems such as coronary heart disease, blindness, kidney failure, stroke and peripheral vascular disease. ${ }^{2-4}$ In fact, hyperglycemia, i.e., consistently high blood glucose levels easily can lead to serious oxidative stress due to the increased production of free radicals. The reactive oxygen and nitrogen species (ROS and RNS) through lipid peroxidation of membrane are responsible for the irreversible changes in the structure of the red blood cell (RBC) cytoskeleton. Therefore, the toxic effect of glucose on erythrocytes manifests itself not only as an alteration of the phospholipids' bilayer but also as a modification of the integral proteins and of the hemoglobin $(\mathrm{Hb})$, protein involved in the oxygen transport, abundantly expressed in the RBCs.

\subsection{Dielectric properties approach}

The study of the dielectric properties of materials can be approached in two ways: (i) a theoretical point of view and (ii) an experimental point of view. The experimental approach is fundamental because it is the first interaction with the medium and it furnishes a quantitative and qualitative vision of the phenomena object of study which occur in the medium and the evolution of these phenomena. This information is utilized to formulate models which are in agreement with experimental data. Obviously for the formulation of these models it is necessary to identify the variables which describe the phenomena. Unfortunately, the number of variables that describe a biological system is very large, so it is essential to reduce this number to formulate a concrete model. Obviously this means substituting the real system with an ideal one whose description is limited to the chosen variables. But, as a consequence of the complexity of biological phenomena, it is very difficult to identify the variables giving the best - or at least a sufficiently good - description. Every model will be associated to a particular choice of variables so to conceive the theoretical approach. From a dielectric point of view, well-known models are based on the introduction of combination in series or in parallel of capacitance and conductance. We call the models based on these combinations classical models. These electrical models, in some cases, describe well the real biological elements and

This is an Open Access article published by World Scientific Publishing Company. It is distributed under the terms of the Creative Commons Attribution 3.0 (CC-BY) License. Further distribution of this work is permitted, provided the original work is properly cited. 
their functions; for example, it is well known that some aspects of the cell membrane are well described by a capacitance element. ${ }^{5,6}$

In this context, the classical models are used to determine dynamical constitutive differential equations for dielectric relaxation which describe some specific properties of the medium under consideration. Of course, depending on the analyzed medium, a suitable combination of capacitance and conductance can be chosen. These models are able to describe some dielectric relaxation phenomena in the linear approximation. $^{7}$

But in the differential equations of these models appear some parameters which are assumed as constant characteristics of the medium. In other words, it is assumed that these parameters do not depend on the type of perturbation.

For example, if the medium is perturbed by a harmonic electric field of frequency, $\omega$, these parameters remain constant for each frequency. Indeed, since these parameters characterize the medium, they must depend on the type of perturbation. Nevertheless, the assumption of the constancy of these parameters will be an approximation which in some cases results in a good one.

Now, if we consider as known the parameters that appear in the classical differential equation, by assuming as known perturbation an extensive variable as the polarization field (the cause), the integration of the dielectric differential equation leads to the knowledge of the intensive variable of the electric field inside the medium (effect). This approach does not give information on the parameters that appear in the equation, but considering them as known, it is able to give information on the effects (electric field) of evolution of the medium when subject to a known perturbation. By starting from nonequilibrium thermodynamic consideration and by introducing the concept of internal hidden variables associated to the internal degree offreedom, Kluitenberg obtained a dielectric relaxation equation that generalizes the above mentioned classical models. ${ }^{8-10}$

Moreover, we have proved that even fractionary models such as Cole-Cole and others will result as particular case of the extended Kluitenberg's introduced by us. This has been possible because Prof. Kluitenberg proved that the polarization vector can be split into two parts. We have proved, in previous papers, ${ }^{11-15}$ that the parameters called phenomenological and state coefficients, which appear in Kuitenberg's dielectric differential equation, depend on the perturbation and we have expressed them as a function of frequency of an harmonic perturbation by means of the relations, obtained by us, between these parameters and some frequencies depending on moduli which can be directly measured.

Moreover, a very important result of the theory is that the so-called phenomenological and state coefficients are correlated to single phenomena (here we will refer only to dielectric media of order one). So we can identify each coefficient just to one phenomenon. Also in this approach it is assumed that the aforementioned coefficients are constant over time, but, unlike models cited, they may depend on some characteristic of the perturbation acting on the medium. In the case considered by us they may depend on the frequency of the harmonic perturbation acting on the medium. Here, as in the aforementioned classical models, if the phenomenological and state coefficients are known and, for example, the polarization, it is possible by integration of the dielectric equation to obtain the behavior of electric field.

In previous papers, ${ }^{11-15}$ we approached the study of dielectric properties of a medium by another point of view: we investigated the behavior of phenomenological and state coefficients assuming as known harmonic perturbation and the response of the medium. This leads to the determination of the aforementioned coefficients as function of the frequency of perturbation, as aforementioned. There we have taken into account the linear response theory. This will be clear in the next sections where we will make a summary of the results obtained by us. Here we emphasize that the response of the medium depends on the type of perturbations.

We will remark that most of the classical models (related to combination in series or in parallel of capacitance and conductance) are always formulated to fit experimental data and, if they are not in agreement with experiments, they are mathematically modified to this aim.

In this work, we do not formulate any model to fit data, but we will study some thermodynamic irreversible processes occurring inside the blood by applying some recent results on nonequilibrium thermodynamic theory obtained by us so to determine the thermodynamic function correlated to the phenomena under study. In other words, we will apply the nonequilibrium thermodynamic approach to the study of a biological system such as blood. ${ }^{7-9}$

We are conscious of the difficulties to adapt the nonequilibrium thermodynamic theory (which is based also on a model of inert matter) to the very complex phenomena that occur in blood. Nevertheless, we think that this new approach can produce very important results because it provides, from a thermodynamic point of view, new and more detailed information on some processes which occur inside the medium so to determine some physical entities (the more important of which is the entropy production) which can be used as diagnosis and to study the evolution of some pathologies. In particular, we will use this new approach to deepen the differences between physiological blood (pb) and diabetic blood (db).

Thus, the problem is the applicability of the nonequilibrium thermodynamic to the phenomena which occur inside system under study: the blood. In other words, can the blood model that we consider satisfy the basic axiom of the nonequilibrium thermodynamics? This will be the subject of the next section in which we introduce a blood model and compare it with the basic axioms on local and instantaneous equilibrium of the thermodynamic of irreversible processes.

Unfortunately our results do not have any direct experimental confirmation, because they consist to obtain the 
expression of some fundamental thermodynamic entities as functions of the frequency of perturbation. In particular, we obtained the following results:

1. analytical expression of the polarization vector $\left(P^{(0)}\right.$, $\left.P^{(1)}\right)$

2. analytical expression of the state coefficients $a^{(0,0)}, a^{(1,1)}$ characteristic of the medium.

3 . analytical expression of coefficients $L^{(1,1)}$ and $L^{(0,0)}$ related to irreversible processes due to the displacement current associated to temporal variation of part of polarization $P^{(1)}$ and to temporal variation of vector $P$, respectively.

4. analytical expression of electric field $E^{(1)}$ related to temporal variation of part of polarization $P^{(1)}$.

5. analytical expression of entropy production.

No results of this kind exist in literature.

\subsection{Mathematical model of the blood and basic axiom}

Human blood accounts for $7 \%$ of the body weight, its main roles in the organisms are: it carries the nutrients and molecular oxygen indispensable to the cellular metabolism and at same time it has an important thermoregulatory role. From the electrical point of view, human blood is a very inhomogeneous solution composed of plasma and several kinds of cells; these cells called corpuscles or "formed elements" consist of erythrocytes (RBCs), leukocytes (white blood cells, WBCs) and thrombocytes (platelets). By volume, the RBCs constitute about $45 \%$ of whole blood, the plasma about $54.3 \%$ and WBCs about $0.7 \%$.

RBCs are biconcave disk shaped and small size $(7 \mu \mathrm{m})$; they are highly specialized cells providing as much space for $\mathrm{Hb}(4-5 \mathrm{mM})$ as possible, in fact they have no nucleus, mitochondria or endoplasmic reticulum. $\mathrm{Hb}$ is a globular protein, the main function of which is to transport oxygen from lungs to respiring tissues. WBCs differ from RBCs because they always have a nucleus and their shape is usually spherical. There are two main groups of WBCs: phagocytes and lymphocytes. Phagocytes which destroy invading cells by phagocytosis are characterized by lobed nuclei and granular cytoplasm. Lymphocytes that destroy microorganisms secreting antibodies are smaller than phagocytes and are recognized by large nucleus. In the blood of all vertebrates, the plasma mostly water contains a wide variety of substances such as proteins, cholesterol, hormones, amino acids, glucose, salts, vitamins and antibodies. Among these an important role is carried out by the glucose sugar, selected as central carbohydrate for its functions both metabolic and structural. Its normal concentration is regulated by several hormones but in some metabolic disorders as well as those present in diabetes, glucose concentration in the blood can reach toxic levels responsible for the functional and structural alterations of the cell constituting the hematic tissue.
In the pathology of DM, the presence of additional glucose in the blood alters the physical-chemical properties of the blood such as the electric and dielectric properties. ${ }^{16-19} \mathrm{~A}$ comparative study of the electrical properties of healthy and pathological blood can lead to a better understanding of biological processes linked to diabetes and to the discovery of new methods for early diagnosis. One of the main alterations that occur in the blood in the presence of high glucose concentrations (diabetes disorders) is the glycosylation of proteins. Glycosylation refers to the covalent bonding of glucose to the blood proteins, in fact, glucose is chemically reactive and has a slight reducing capacity to accept electrons from the $\varepsilon$-amino group of a protein lysine residue, to form an adduct commonly referred to as a Schiff base. Lysine is found in virtually every protein and when that electron is shared it creates an irreversible chemical bond between the glucose and the protein. Glycosylation can also occur at the N-terminal amino group of the $\beta$-chains by a ketoamine linkage. This is the case of the mainly glycosylated protein in blood, the $\mathrm{HbA} 1 \mathrm{c}$ is the result of nonenzymatic reaction of glucose with the $\alpha$-amino groups of the Valine residues at the $\mathrm{N}$ terminus of $\mathrm{Hb} \beta$-chains. ${ }^{20}$ Normally, only a small percentage of blood glucose, usually between $4.5 \%$ and $6 \%$, is covalently linked to the $\mathrm{Hb}$ of nondiabetes population. But, under conditions of sustained hyperglycemia such as DM, a significant increase in the percentage of glycosylated $\mathrm{Hb}$ is commonly noted: HbA1c is increased two- to three-fold in the RBCs. Nevertheless the increased amount and permanence of glucose in the blood allows more glycosylation to occur, not only with $\mathrm{Hb}$, but with the proteins of the erythrocyte membrane and with other proteins of the plasma. The binding of glucose, especially with important protein amino groups, can affect cell structure and function and create an unbalance which leads to cell destabilization. Because, each of these proteins can undergo cyclization and rearrangement or cross linking to create something called advanced glycosylation end products (AGEs). ${ }^{21}$

Our approach is based on the assumption (supported by experiments) that in biological tissue hidden electrical phenomena occur to which corresponding internal degree of freedom must be taken into account for a more detailed description and prediction.

So we consider blood as a conducting fluid in which three major micro-particles are suspended (RBCs, WBCs, MLPs) and distributed in an homogeneous way. ${ }^{5,6,22}$

This allows us to consider blood as a continuum medium which obeys the continuum mechanics indefinite equations and Maxwell's equation in the matter with a mass density $\rho$ which varies as a function of blood's elements under consideration. ${ }^{23}$ In Sec. 2.3, where we describe in a synthetic form our theory, we will prove that, by considering blood as an incompressible fluid, the Lagrangian's derivative $\frac{d \rho}{d t}$ of the mass density results in zero. In other words, we will prove that the mass density is almost constant for each fluid element during the motion. This is justified if we consider the mass 
density of three major micro-particles of blood (as RBCs, WBCs, MLPs).

Such an assumption is also in agreement with the basic axiom on local and instantaneous equilibrium: for sufficiently small deviation from equilibrium, a system can be divided into tiny (physical) volume elements, each of which can be regarded as a small homogeneous equilibrium system.

Moreover, the length and time scale of these subsystems are infinitesimally small from a macroscopic point of view, but from a molecular point of view they are still large, such that the subsystem contains enough molecules so that the average taken on the number of molecules has deterministic significance.

Since we will study blood when it is subject to an external harmonic electric perturbation we assume that in this case the axiom of local and instantaneous equilibrium will be satisfied; this is true for a sufficiently low intensity perturbation.

\section{Methods}

\subsection{Theoretical thermodynamic approach}

Kluitenberg's theory is based on the idea that the usual variables of nonequilibrium thermodynamic are insufficient to describe some phenomena that occur in a medium when it is subject to perturbation. In particular, they are insufficient to describe relaxation dielectric phenomena in a continuous media (we neglect the magnetic effects).

Generally it is assumed that the specific entropy $s$ of an elastic dielectric is a function of the specific internal energy $u$, the strain tensor $\varepsilon_{i k}$ and the specific polarization $p^{8-10}$ :

$$
s=s\left(u\left|\varepsilon_{i k}\right| \underline{p}\right) .
$$

The new Kluitenberg's idea consists in the assumption that there is a vector field $\Omega$ which plays the role of thermodynamic internal degree of freedom and which influences the polarization. ${ }^{8-10}$ In the theory it is assumed that the specific entropy (which we indicate with $s$ ) has the following functional dependence

$$
s=s\left(u\left|\varepsilon_{i k}\right| \underline{p} \mid \underline{\Omega}\right) .
$$

From which it follows:

$$
\left\{\begin{array}{l}
\frac{1}{T}=\frac{\partial s\left(u\left|\varepsilon_{i k}\right| \underline{p} \mid \underline{p}^{(1)}\right)}{\partial u}, \\
\tau_{i k}^{(\mathrm{eq})}=-T \rho \frac{\partial s\left(u\left|\varepsilon_{i k}\right| \underline{p} \mid \underline{p}^{(1)}\right)}{\varepsilon_{i k}}, \\
\underline{E}^{(\mathrm{eq})}=-T \frac{\partial s\left(u\left|\varepsilon_{i k}\right| \underline{p} \mid \underline{p}^{(1)}\right)}{\partial \underline{p}}, \\
\underline{E}^{(1)}=T \frac{\partial s\left(u\left|\varepsilon_{i k}\right| \underline{p} \mid \underline{p}^{(1)}\right)}{\partial \underline{p}^{(1)}} .
\end{array}\right.
$$

By assuming that exists a state $R$ at the constant temperature $T_{0}$ in which it results:

$$
\begin{aligned}
& \tau_{i k}^{(\mathrm{eq})}\left(T_{0}\right)=\tau_{i k(0)}^{(\mathrm{eq})}=0, \\
& \underline{E}^{(\mathrm{eq})}\left(T_{0}\right)=\underline{E}_{(0)}^{(\mathrm{eq})}=0
\end{aligned}
$$

it can be shown that the polarization $p$ is additively composed of two parts $\underline{p}^{(0)}$ and $\underline{p}^{(1)}$

$$
\underline{p}=\underline{p}^{(0)}+\underline{p}^{(1)},
$$

where following the Debye's model, the vector $\underline{p}^{(0)}$ is associated with molecule deformation polarization and $p^{(1)}$ is associated with molecule rotation polarization.

Moreover, it can be shown that the change of both $p^{(0)}$ and $p^{(1)}$ contributes to entropy production and therefore they represent two irreversible processes. With these clarifications, Eq. (2a) can be written as:

$$
s=s\left(u\left|\varepsilon_{i k}\right| \underline{p} \underline{p}^{(1)}\right)
$$

and introduction of vector $\underline{E}^{(\text {ir) }}$ defined as:

$$
\underline{E}^{(\mathrm{ir})}=\underline{E}-\underline{E}^{(\mathrm{eq})}
$$

is possible, where $\underline{E}$ is the electric field which occurs in Maxwell's equations and $\underline{E}^{(i r)}$ is called the irreversible electric field. If the irreversible electric field (4) vanishes the change in $p^{(0)}$ does not contribute to the entropy production, i.e., changes in $p^{(0)}$ are reversible processes. ${ }^{10}$

\subsection{Phenomenological equations}

The entropy production per unit of volume and per unit of time is given by ${ }^{10}$

$$
\begin{aligned}
\sigma^{(s)}= & \underline{J}^{(Q)} \cdot \underline{X}^{(Q)}+\underline{J}^{(\mathrm{el})} \underline{E} \\
& +\rho \underline{E}^{(\mathrm{ir})} \frac{d \underline{p}}{d t}+\rho \underline{E}^{(1)} \frac{d \underline{p}}{d t},
\end{aligned}
$$

where $\underline{J}^{(Q)}$ is the heat flow and $\underline{J}^{(\mathrm{el})}$ is the density of electric current;

$$
\underline{X}^{(Q)}=-T^{-1} \operatorname{grad} T .
$$

It appears that the entropy production (5) is due to heat conduction, electric conduction and dielectric relaxation. Moreover, it is a sum of inner products of vectors.

In agreement with the method of nonequilibrium thermodynamics developed by De Groot e Mazur (see Ref. 7), there will be linear relations among these quantities which, for an isotropic media, can be written as:

$$
\begin{gathered}
\underline{E}^{(\mathrm{ir})}=\rho L^{(0,0)} \frac{d \underline{p}}{d t}+L^{(0,1)} \underline{E}^{(1)}, \\
\rho \frac{d \underline{p}^{(1)}}{d t}=\rho L^{(1,0)} \frac{d \underline{p}}{d t}+L^{(1,1)} \underline{E}^{(1)},
\end{gathered}
$$


where $L^{(0.0)}, L^{(0.1)}, L^{(1.0)}, L^{(1.1)}, L^{(0,1)}, L^{(1,0)}$ are phenomenological coefficients and we shall assume that they are constant in time. The coefficients $L^{(0.1)}, L^{(1.0)}$ are connected with possible cross effects which may occur between the two types of dielectric relaxation phenomena described by Eqs. (6) and (7), and they satisfy the Onsager-Casimir reciprocal relations

$$
L^{(0.1)}=-L^{(1.0)}
$$

If we neglect the cross effect described by coefficients $L^{(0.1)}$ and $L^{(1.0)}$ we observe that, the coefficient $L^{(0.0)}$, which has the dimension of a resistance, is connected to irreversible processes related to change of $p$ (and therefore of $p^{(0)}, p^{(1)}$ or both $p^{(0)}$ and $p^{(1)}$, while $L^{(1.1)}$, which has the dimension of a conductibility, is related to change of $p^{(1)}$ and the corresponding intensive variable $\underline{E}^{(1)}$. However, Eqs. (6) and (7) are connected with irreversible changes in the polarization.

\subsection{Linear approximation state equations}

The free energy $f$ is defined by

$$
f=u-T s .
$$

It can be shown that there exists the following functional dependence of $f$ :

$$
f=f\left(T\left|\varepsilon_{i k}\right| \underline{p} \mid \underline{p}^{(1)}\right)
$$

This is the specific free energy and it can be shown ${ }^{8-10}$ that the condition of isotropy and of linearity of equations of state are fulfilled if it is assumed that $f$ is the sum of two functions $f_{1}$ and $f_{2}$

$$
f=f_{1}+f_{2},
$$

where

$$
\begin{gathered}
f_{1}=f_{1}\left(T \mid \varepsilon_{i k}\right), \\
f_{2}=\frac{1}{2} \rho\left[a^{(0,0)} \underline{p} \cdot\left(\underline{p}-2 \underline{p}^{(1)}\right)+a^{(1,1)} \underline{p}^{(1)^{2}}\right],
\end{gathered}
$$

where $a^{(0,0)}$ and $a^{(1,1)}$ are state coefficients (assumed constant) which have the dimension of reciprocal dielectric constant.

By defining the fields $\underline{P}^{(0)}$ and $\underline{P}^{(1)}$ by

$$
\begin{gathered}
\underline{P}^{(0)}=\rho \underline{p}^{(0)}, \\
\underline{P}^{(1)}=\rho \underline{p}^{(1)}, \\
\underline{P}=\underline{P}^{(0)}+\underline{P}^{(1)},
\end{gathered}
$$

we can obtain from equation, the following state equations

$$
\begin{gathered}
\underline{E}^{(\mathrm{eq})}=a^{(0,0)}\left(\underline{P}-\underline{P}^{(1)}\right)=a^{(0,0)} \underline{P}^{(0)}, \\
\underline{E}^{(1)}=a^{(0,0)} \underline{P}-a^{(1,1)} \underline{P}^{(1)},
\end{gathered}
$$

which together with Eqs. (6) and (7), in which we neglect the cross effects which may occur between them,

$$
\begin{gathered}
\underline{E}^{(\mathrm{ir})}=L^{(0,0)} \frac{d \underline{P}}{d t}, \\
\frac{d \underline{P}^{(1)}}{d t}=L^{(1,1)} \underline{E}^{(1)} \\
=L^{(1,1)}\left[\underline{E}^{(\mathrm{eq})}+\left(a^{(0,0)}-a^{(1,1)}\right) \underline{P}^{(1)}\right]
\end{gathered}
$$

will be the equations that allow us to the introduction of relaxation equation.

As aforementioned now we prove that for a fluid as blood it is reasonable to assume that $\rho$ is constant for each element so to verify the basic axioms on local and instantaneous equilibrium.

In fact, the assumption that the blood is an incompressible fluid is expressed for a fluid's element $d \tau$ by mathematical relation $\frac{d}{d t}(d \tau)=0$; so, from the well-known cinematic relation $\frac{d}{d t}(d \tau)=\operatorname{div} \underline{V} d \tau$, it follows $\operatorname{div} \underline{V}=0$. This last equation and the equation of conservation of the mass $\frac{d \rho}{d t}+$ $\rho \operatorname{div} \underline{V}=0$ lead to assert that.

$$
\frac{d \rho}{d t}=\frac{\partial \rho}{\partial t}+\underline{V} \cdot \operatorname{grad} \rho=0 .
$$

Here $\underline{V}$ is the velocity of the fluid element. If the fluid is not homogenous at the initial instant its initial density $\rho_{0}$ varies with Lagrangian' coordinates $\left(b_{1}, b_{2}, b_{3}\right)$ so as to have

$$
\rho_{0}=\rho_{0}\left(b_{1}, b_{2}, b_{3}\right) \text {. }
$$

By substituting the following relation (which is valid during the motion) $b_{i}=b_{i}\left(t, x_{1}, x_{2}, x_{3}\right)$ in the last equation, one has

$$
\rho_{0}\left(b_{1}, b_{2}, b_{3}\right)=\rho_{0}\left[b_{i}\left(t, x_{i}\right)\right]=\rho\left(t, x_{1}, x_{2}, x_{3}\right) .
$$

This relation satisfy Eq. (15) and proves the assert.

Thus we assume that the mass density $\rho$ is constant. ${ }^{8-10}$ It is seen from Eq. (14) that sudden change in $\underline{P}^{(1)}$ is impossible, while from Eq. (13) it follows that sudden change in $\underline{P}^{(0)}$ is possible.

\subsection{Relaxation equation}

In the following, we assume that $T=$ constant, in agreement with blood status. It can be shown that it is possible to eliminate the internal fields and the two fields $\underline{P}^{(0)}$ and $\underline{P}^{(1)}$ from Eqs. (11)-(14) so to obtain the so-called relaxation equation, mentioned in Sec. 1:

$$
\chi_{E P}^{(0)} \underline{E}+\frac{d \underline{E}}{d t}=\chi_{P E}^{(0)} \underline{P}+\chi_{P E}^{(1)} \frac{d \underline{P}}{d t}+\chi_{P E}^{(2)} \frac{d^{2} \underline{P}}{d t^{2}},
$$

where

$$
\begin{gathered}
\chi_{E P}^{(0)}=a^{(1,1)} L^{(1,1)}, \\
\chi_{P E}^{(0)}=a^{(0,0)}\left(a^{(1,1)}-a^{(0,0)}\right) L^{(1,1)}, \\
\chi_{P E}^{(1)}=a^{(0,0)}+a^{(1,1)} L^{(0,0)} L^{(1,1)},
\end{gathered}
$$




$$
\chi_{P E}^{(2)}=L^{(0,0)}
$$

Moreover it can be shown that phenomenological and state coefficients together with $\chi_{E P}^{(0)}, \chi_{P E}^{(0)}, \chi_{P E}^{(1)}, \chi_{P E}^{(2)}$ must be positive in order to satisfy the principle of entropy production. Moreover, the following relations must be satisfied:

$$
\begin{aligned}
& \chi_{P E}^{(1)}-\chi_{E P}^{(0)} \chi_{P E}^{(2)}>0, \\
& \chi_{P E}^{(1)} \chi_{E P}^{(0)}-\chi_{P E}^{(0)} \geq 0 .
\end{aligned}
$$

The importance of the phenomenological and state coefficients is that they characterize the medium specifying the amount of type of phenomena correlating to each of them.

It is important to observe that their constancy is referred to the time for each type of perturbation which acts on the medium. But they vary with the change of perturbation. For example, if the perturbation is of harmonic type with frequency $\omega$ then the coefficients will depend on $\omega$ (we will see this in next section) which can be considered as parameter in the functional dependence of the coefficients. In this case, we shall call $a^{(0,0)}, a^{(1,1)}, L^{(0,0)}, L^{(1,1)}$ dynamical coefficients.

\subsection{Phenomenological and state coefficients as function of frequency}

In the following, we consider the vector component normal to the surface of the electrode, the latter being on the surface of blood. Assuming that $P$ varies as

$$
P=P_{0} \sin \omega t
$$

and by introducing some appropriate approximations, phenomenological and state coefficients can be expressed as functions of the frequency as follows ${ }^{15,23}$ :

$$
\begin{gathered}
a^{(0,0)}(\omega)=\Gamma_{1}+\frac{\Gamma_{2}^{(1)}}{\omega \sigma}, \\
a^{(1,1)}(\omega)=\frac{\left[\Gamma_{2}^{(1)}+\Gamma_{1} \omega \sigma\right]^{2}}{\omega \sigma \Gamma_{2}^{(1)}\left(1+\omega^{2} \sigma^{2}\right)}, \\
L^{(1,1)}(\omega)=\frac{\omega \Gamma_{2}^{(1)}\left(1+\omega^{2} \sigma^{2}\right)}{\left[\Gamma_{2}^{(1)}+\Gamma_{1} \omega \sigma\right]^{2}}, \\
L^{(0,0)}(\omega)=\frac{\Gamma_{2 R}}{\omega},
\end{gathered}
$$

where

$$
\begin{aligned}
& \Gamma_{1}=\frac{\varepsilon_{1}-\varepsilon_{0}}{\left(\varepsilon_{1}-\varepsilon_{0}\right)^{2}+\varepsilon_{2}^{2}}, \\
& \Gamma_{2}=\frac{\varepsilon_{2}}{\left(\varepsilon_{1}-\varepsilon_{0}\right)^{2}+\varepsilon_{2}^{2}}
\end{aligned}
$$

and $\Gamma_{2 R}$ is the relaxed value for $\omega=\omega_{R}{ }^{24}$ Here $\omega_{R}$ is the frequency under which $\Gamma_{2}$ is almost constant.

Here $\varepsilon_{1}$ and $\varepsilon_{2}$ are real and imaginary parts, respectively, of the complex dielectric constant and $\sigma$ is the relaxation time. Here, we introduced a new quantity defined as:

$$
\Gamma_{2}^{(1)}=\Gamma_{2}-\omega L^{(0,0)} \text {. }
$$

By remembering the meaning of $\Gamma_{2},{ }^{24}$ the last equation represent the difference between the total loss modulus and the loss associated to $E^{(\mathrm{ir})}$ (then $E^{(\mathrm{ir})}$ and $L^{(00)}$ are not related to internal degree of freedom); in other words Eq. (27) represents the dissipation associated to internal degree of freedom.

From (22) and (24), it follows that, if $\Gamma_{2}^{(1)}=0$, no dissipation is connected to internal degree of freedom, it follows:

$$
\begin{gathered}
a^{(0,0)}(\omega)=\Gamma_{1}, \\
L^{(1,1)}=0 .
\end{gathered}
$$

And from (14) and (24) it follows

$$
P^{(1)}=\text { cost. }=0 \text {. }
$$

We observe that $\Gamma_{2}=\omega L^{(00)}$ if $\Gamma_{2}^{(1)}=0\left(L^{(11)}=0\right)$; this occurs for sufficiently low frequency where the internal degree of freedom does not appear. So we can put ${ }^{15}$

$$
\Gamma_{2}(\text { low frequency })=\Gamma_{2 R}=\omega L^{(0,0)}
$$

2.6. Evaluation of the fields $E^{(\mathrm{eq})}, E^{(1)} E^{(\mathrm{ir})}, P^{(0)}, P^{(1)}$

Now we will evaluate the fields $\underline{E}^{(\mathrm{eq})}, \underline{E}^{(1)} \underline{E}^{(\mathrm{ir})}, \underline{P}^{(0)}, \underline{P}^{(1)}$ taking into account Eqs. (22)-(25). This allows us an experimental evaluation of these fields since are known by experimental measurements coefficients (22)-(25).

We introduce the normal component of $\underline{E}^{(\mathrm{eq})}, \underline{E}^{(1)} \underline{E}^{(\mathrm{ir})}$, $\underline{P}^{(0)}, \underline{P}^{(1)}$ which we will indicate as $E^{(\mathrm{eq})}, E^{(1)} E^{(\mathrm{ir})}, \bar{P}^{(0)}, \underline{P}^{(1)}$.

In agreement with linear response theory, ${ }^{23,24}$ we assume that $P$ varies as Eq. (21), and that $E$ varies as

$$
E=E_{0}(\omega) \sin (\omega t+\phi(\omega))
$$

or

$$
E=P_{0} \Gamma_{1} \sin \omega t+P_{0} \Gamma_{2} \cos \omega t,
$$

where

$$
\left\{\begin{array}{l}
\Gamma_{1}=\frac{E_{0}(\omega)}{P_{0}} \cos \phi(\omega), \\
\Gamma_{2}=\frac{E_{0}(\omega)}{P_{0}} \sin \phi(\omega) .
\end{array}\right.
$$

$\phi(\omega)$ is the phase lag between $P$ and $E$. From this it follows that the relaxed value $\Gamma_{1 R}$ obtained for $\omega=\omega_{R}$ will be

$$
\Gamma_{1 R}=\frac{E_{0 R}}{P_{0}},
$$

where

$$
E_{0 R}=E\left(\omega=\omega_{R}\right) .
$$


Combining (33) and (34) it follows

$$
E_{0}(\omega)=\frac{\Gamma_{1}}{\cos \phi(\omega)} P_{0}=\frac{\Gamma_{1}}{\cos \phi(\omega)} \frac{E_{0 R}}{\Gamma_{1 R}} .
$$

Now we are able to obtain an explicit form of the aforementioned fields which is suitable for experimental evaluation.

From Eqs. (13) and (21) it follows:

$$
E^{(\mathrm{ir})}=L^{(0,0)} \frac{d P}{d t}=L^{(0,0)} \frac{E_{0 R}}{\Gamma_{1 R}} \omega \cos \omega t .
$$

From (32), (36) and (37) it follows

$$
E=\Gamma_{1} P+\frac{\Gamma_{2}}{L^{(0,0)} \omega} E^{(\mathrm{ir})} .
$$

This equation is similar to Eq. (4). They can be equated if $\Gamma_{2}$ is connected only with irreversible dielectric relaxation phenomena, since in general it is the synthesis of many dissipative phenomena which occur inside the medium. We impose this equality assuming for $\Gamma_{2}$ the value $\Gamma_{2}\left(\omega_{R}\right)=\Gamma_{2 R}$ for low (and high) frequency which we consider as the "best representation" of the aforementioned phenomena (in analogy with mechanical case). In this case we have:

$$
\begin{aligned}
& E^{(\mathrm{eq})}=\Gamma_{1} P, \\
& L^{(0,0)}=\frac{\Gamma_{2 R}}{\omega} .
\end{aligned}
$$

From (11) and (39) it follows

$$
P^{(0)}=\frac{\underline{E}^{(\mathrm{eq})}}{a^{(0,0)}}=\frac{\Gamma_{1} P}{a^{(0,0)}},
$$

where $a^{(00)}$ is given by Eq. (22).

From (2b), (21) and (41) it follows:

$$
P^{(1)}=P-P^{(0)}=P\left(1-\frac{\Gamma_{1}}{a^{(0,0)}}\right) .
$$

By taking into account (14) and (42) one has:

$$
E^{(1)}=\frac{1}{L^{(1,1)}} \frac{d P^{(1)}}{d t}=\frac{P_{0} \omega \cos \omega t}{L^{(1,1)}}\left(1-\frac{\Gamma_{1}}{a^{(0,0)}}\right) .
$$

The entropy production (5) in the case of only dielectric relaxation phenomena becomes:

$$
\sigma^{(s)}=T^{-1}\left(E^{(\mathrm{ir})} \frac{d p}{d t}+E^{(1)} \frac{d p^{(1)}}{d t}\right),
$$

where heat flow and density of electric current have been neglected.

Taking into account Eqs. (21), (30b), (42) and (43) one has:

$$
\sigma^{(s)}=\frac{P_{0}^{2}}{T}\left(\omega L^{(0,0)}+\frac{\Gamma_{2}^{(1)}}{\left(1+\omega^{2} \sigma^{2}\right)}\right) \omega \cos ^{2} \omega t .
$$

\section{Results and Conclusions}

Here we will refer to experimental data obtained by Abdalla et al. ${ }^{5}$ for complex dielectric constant of the $\mathrm{pb}$ and $\mathrm{db}$. We use these data for calculation of thermodynamic functions of the theory and we analyze the results obtained following the Debye's point of view ${ }^{8-10}$ according to which it is assumed that for molecule deformation polarization is associated with vector $P^{(0)}$ and for molecule rotation polarization is associated with $\underline{P}^{(1)}$. Obviously, for each molecule both phenomena may be present.

Here as aforementioned, we consider only the component of the vector that is normal to the surface plate, so we write $P^{(0)}, P^{(1)}$ for $\mathrm{pb}$ and $P_{d}^{(0)}, P_{d}^{(1)}$ for $\mathrm{db}$. In general, we will indicate with an index " $d$ " the function referred to $\mathrm{db}$.

We start by analyzing the behavior of $P^{(0)}$ both for $\mathrm{pb}$ and for $\mathrm{db}$. By observing Fig. 1 we note that by increasing frequency it results always in $P^{(0)}>P_{d}^{(0)}$. This means that in $\mathrm{pb}$ we have a greater number of dipoles that follow the perturbation field than in $\mathrm{db}$. From a biochemical point of view we explain this by observing that excess glucose in $\mathrm{db}$ will lead to generation of chemical bonds between blood elements and sugar.

In this context, the glycosylation of proteins may be the cause of reduced movement of elongation. In fact, glucose reacting nonenzymatically with proteins to form stable covalent linkages increases the molecular weight of glycated proteins. ${ }^{25}$ Besides, further increases in blood sugar lead to further glycation of proteins and lipids with molecular rearrangements that lead to generation of AGEs.

Other intracellular and membrane proteins of RBCs are also glycated, for example Spectrin, a major RBC membrane protein, band 3 transmembrane protein, and band 4-1. The glycation results in reduced $\mathrm{RBC}$ deformability and in an increased adherence to endothelium. Platelet membrane proteins can be glycated. The increased binding of fibrinogen and the increased platelet aggregation observed in diabetic patients ${ }^{26}$ can be related to glycation of the adenosine diphosphate receptors and lipids indicating that the number of

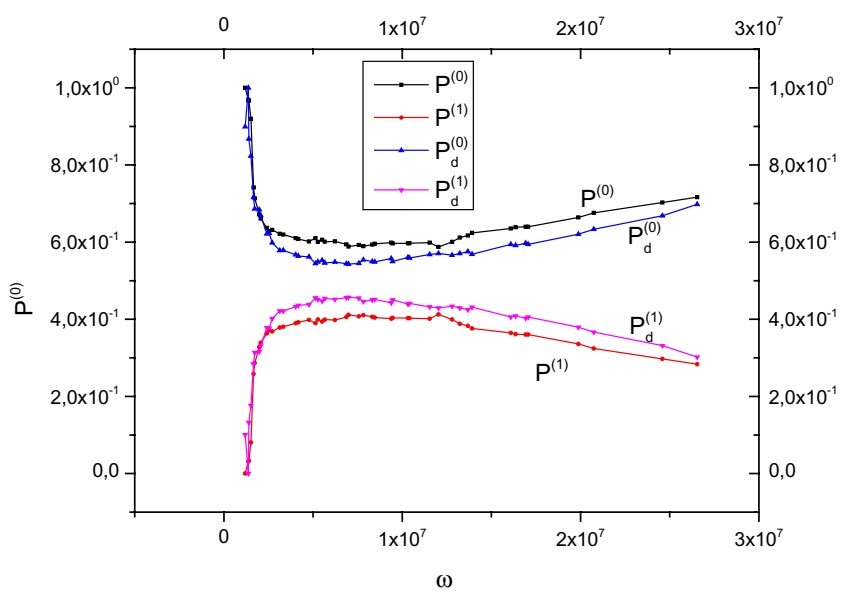

Fig. 1. $P^{(0)}$ and $P^{(1)}$ for $\mathrm{pb}$ and db (Eq. (42)). 


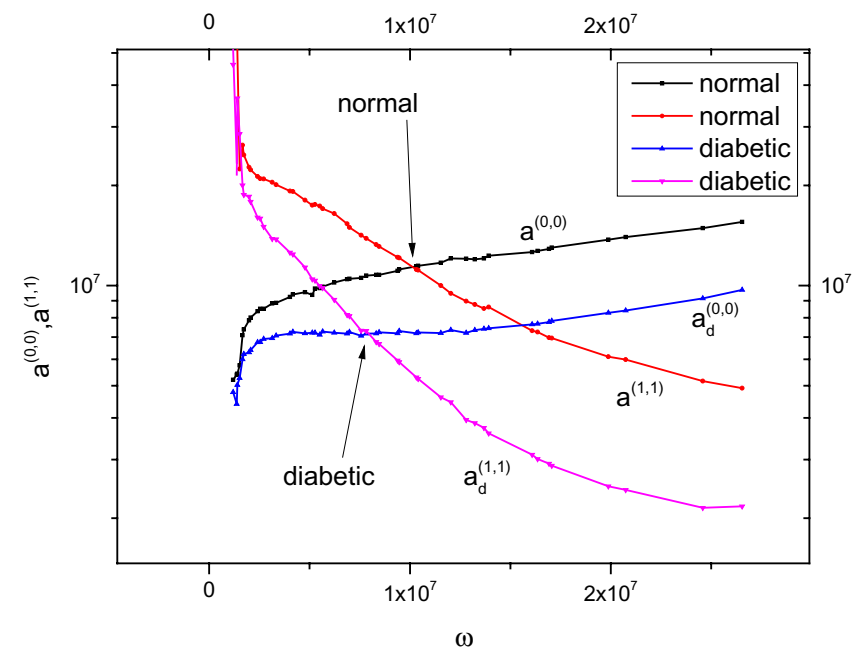

Fig. 2. State coefficients for pb and db (Eqs. (22) and (23)).

dipoles that follows the field is larger when the frequency increases. In other words, we have a new generation of polarization dipoles when frequency increases. Obviously the field $E^{(\mathrm{eq})}=a^{(0,0)} P^{(0)}$ (see Eq. (11)) will be an increasing function, as confirmed by Fig. 4, since this occurs for the coefficient $a^{(0,0)}$ (see Fig. 2): the blood will be structured in such a way that in every point the field associated to polarization $P^{(0)}$ (for deformation) will increase. By invoking the same motivation used for explaining $P^{(0)}>P_{d}^{(0)}$ it is easy to explain the relation $E^{(\mathrm{eq})}>E_{d}^{\text {(eq) }}$.

Now we analyze the polarization $P^{(1)}$. On contrary to $P^{(0)}$ it results in $P_{d}^{(1)}>P^{(1)}$ as shown in Fig. 1 and moreover $P_{d}^{(1)}$, $P^{(1)}$ are decreasing functions of the perturbation frequency. By invoking the meaning associated to $P^{(1)}$ we can assert that when the perturbation frequency increases we have a lower number of permanent dipoles that follow the perturbation field. From a biochemical point of view we explain that $P_{d}^{(1)}$ $>P^{(1)}$ by observing that the glucose bonding with the blood elements will form a greater number of permanent dipoles with respect to $\mathrm{pb}$. In particular, as a result of nonenzymatic glycosylation a bulky glycogroup is introduced onto a free amino group leading to a change in the isoelectric point (pI) of the proteins. The alteration in $\mathrm{pI}$ will be more pronounced if the sugar is phosphorylated. In fact, as it is known, the glucose within the cell is immediately phosphorylated by the action of the kinase. The latter, having a low $K_{M}$ for the substrate catalyzes the reaction that leads to the formation of the sugar phosphoric ester. In this way, in addition to neutralization of positive charge on $\varepsilon \mathrm{NH}_{3}^{+}$of Lys (glycosylation), two negative charges are added on the protein due to ionization of phosphate linked to carbon 6 of glucose. This change in electric charge of protein may facilitate oscillation in the field. Not to be neglected is also that all the Lys residues of proteins are susceptible to glycosylation and that the variation of electric charge is proportional to the number of Lys characterizing each protein.
Here we will emphasize that, even if we can separate, both deformation and rotation of polarization are present in each blood element as it is shown by Eq. (12) if we rewrite it in the form:

$$
E^{(1)}=a^{(0,0)} P^{(0)}+\left(a^{(0,0)}-a^{(1,1)}\right) P^{(1)}=E^{(\mathrm{eq})}+E_{x}^{(1)},
$$

where

$$
E_{x}^{(1)}=\left(a^{(0,0)}-a^{(1,1)}\right) P^{(1)}
$$

is the field associated only to rotation polarization of the permanent dipoles represented by $P^{(1)}$. From Eq. (46) we can deduce that the field $E^{(1)}$ is sum of two contributes: $E^{(\mathrm{eq})}$ associated only to $P^{(0)}$ (see Eq. (39)) and $E_{x}^{(1)}$ associated only to $P^{(1)}$ (see Eq. (47)). In other words, while to the generation of the field $E^{\text {(eq) }}$ contributes only the polarization for deformation, to the generation of the field $E^{(1)}$ contributes both the polarization for deformation and for orientation (see Eq. (46)). As for $E^{(\mathrm{eq})}$ we can analyze the field $E_{x}^{(1)}$ associated only to polarization $P^{(1)}$ for both $\mathrm{pb}$ and $\mathrm{db}$; by observing Fig. 3 we note that there exists a frequency in which it results:

$$
E_{x}^{(1)}=E^{(1)}-E^{(\mathrm{eq})}=\left(a^{(0,0)}-a^{(1,1)}\right) P^{(1)}=0
$$

for both $\mathrm{pb}$ and $\mathrm{db}$. This does not means that $P^{(1)}=0\left(P^{(1)}\right.$ is never zero, see Fig. 1) but that $a^{(0,0)}=a^{(1,1)}$ as it is shown by Fig. 2. In fact, at the same frequency in which it results $E_{x}^{(1)}=0$ we have $a^{(0,0)}=a^{(1,1)}$. This is true for both $\mathrm{pb}$ and $\mathrm{db}$, so we may write:

$$
\begin{array}{r}
E_{x}^{(1)}=\left(a^{(0,0)}-a^{(1,1)}\right) P^{(1)}=0 \Rightarrow a^{(0,0)}-a^{(1,1)}=0 \\
\text { for } \omega=\omega_{x}, \\
E_{x d}^{(1)}=\left(a_{d}^{(0,0)}-a_{d}^{(1,1)}\right) P_{d}^{(1)}=0 \Rightarrow a_{d}^{(0,0)}-a_{d}^{(1,1)}=0 \\
\text { for } \omega=\omega_{x d} .
\end{array}
$$

This is shown in Figs. 2 and 3. It is easy to show that from Eqs. (22) and (23) it results $a^{(0,0)}=a^{(1,1)}$ if $\Gamma_{2}^{(1)} \omega \sigma=\Gamma_{1}$.

We note that (see Figs. 2 and 3 ) it results

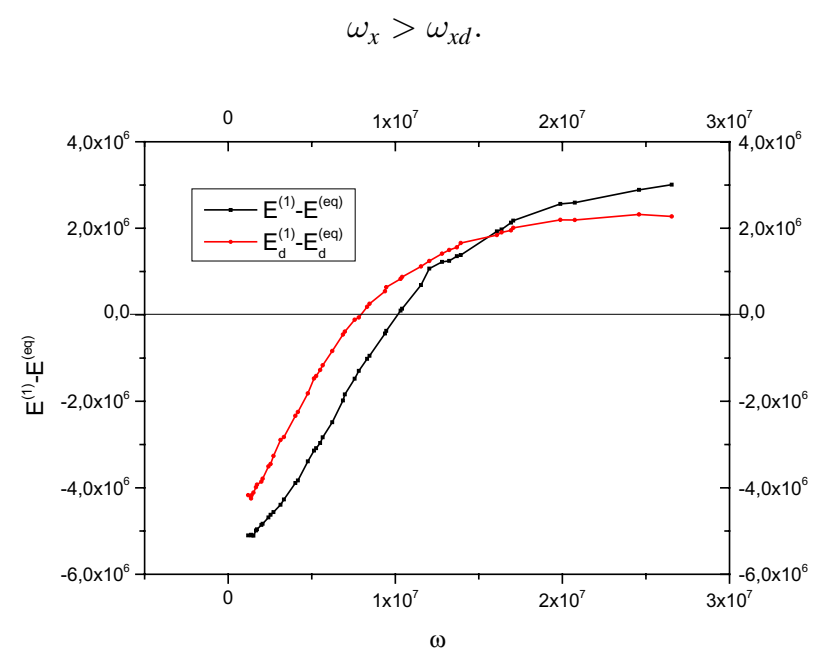

Fig. 3. Field associated only to rotation polarization for $\mathrm{pb}$ and $\mathrm{db}$ (Eq. (46)). 


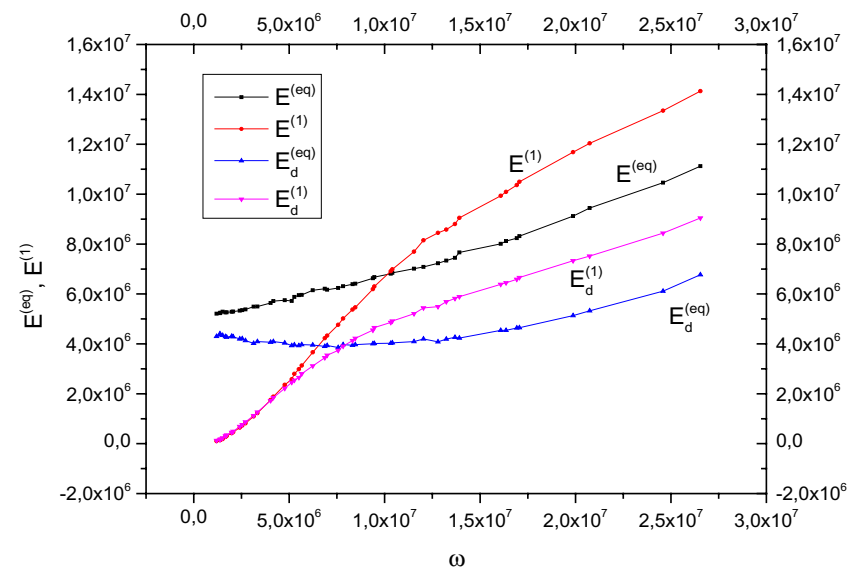

Fig. 4. $E^{(\mathrm{eq})}$ and $E^{(1)}$ electric field for pb and db (Eqs. (11) and (12)).

This is a very important result: the existence of a frequency in which it results $E_{x}^{(1)}=0$ or $a^{(0,0)}=a^{(1,1)}$ is, in general, a peculiarity of blood, but we observe that in this case frequency depends on the level of glucose in blood. In particular, glucose and $\omega_{x d}$ values are linked by an inverse correlation. This correspondence between frequency and glucose level may be utilized as an alternative diagnostic method to measure the values of sugar in blood. In fact, the glucose concentration is easily obtained determining the value of frequency for which $a^{(0,0)}=a^{(1,1)}$.

By observing Fig. 4 we note that

$$
\begin{aligned}
& \text { for } \omega<\omega_{x} \text { it results } E^{(\mathrm{eq})}>E^{(1)} \\
& \quad \text { and for } \omega>\omega_{x} \text { it results } E^{(\mathrm{eq})}<E^{(1)},
\end{aligned}
$$

i.e., the difference $E^{(1)}-E^{(\mathrm{eq})}$ changes the sign in $\omega_{x}$. The frequency $\omega_{x}$ is very important because it is a ring of union between two states of blood (diabetic or not), unfortunately we are not still able to give a biological explication of this change. This change is well represented by Eq. (46) and it is shown in Fig. 5.

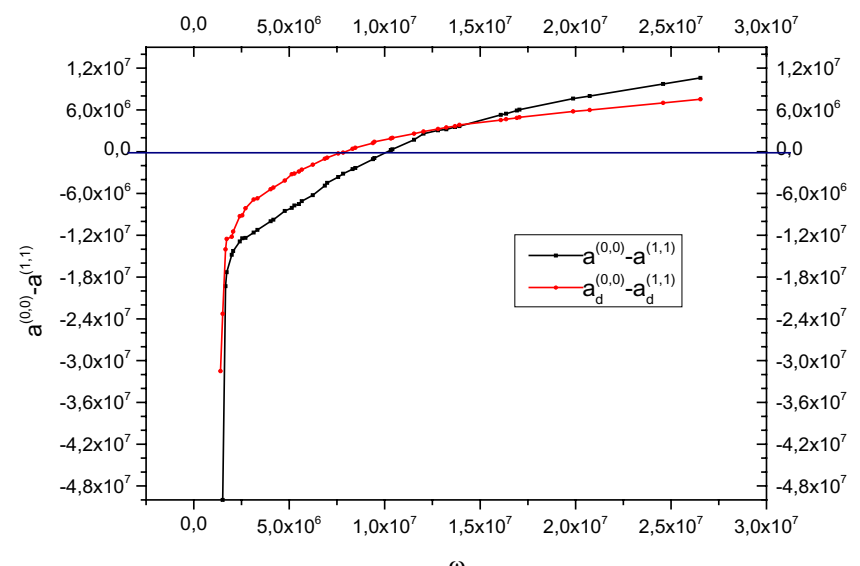

Fig. 5. Difference between state coefficients for pb and db (Eqs. (22) and (23)).

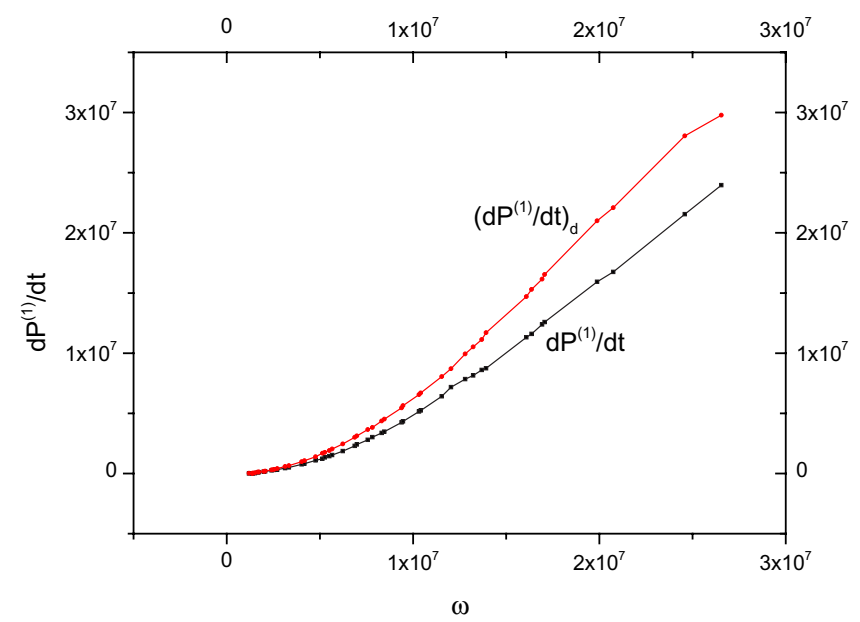

Fig. 6. Displacement current associated to rotation polarization for $\mathrm{pb}$ and $\mathrm{db}$ (Eq. (43)).

As regards the displacement current $d P^{(1)} / d t$ associated to rotation polarization, we note in Figs. 6 and 7 that they show an increment trend for both $\mathrm{db}$ and $\mathrm{pb}$ with greater values for $\mathrm{db}$ with respect to $\mathrm{pb}$. In other words, a temporal change in $P^{(1)}$ will result greater in $\mathrm{db}$ than in $\mathrm{pb}$. This means that some molecules in db have a greater speed of oscillation then in the $\mathrm{pb}$. We may explain this by assuming a greater polarity in $\mathrm{db}$ (see Fig. 1).

Examining the entropy production we note that over a frequency (see Fig. 8), which we indicate with $\omega_{s} \cong 10^{7} \mathrm{~Hz}$, the entropy production will result greater in $\mathrm{pb}$ than in $\mathrm{db}$.

$$
\begin{array}{ll}
\sigma_{d}^{(s)}>\sigma^{(s)} & \text { if } \omega<\omega_{s}, \\
\sigma_{d}^{(s)}<\sigma^{(s)} & \text { if } \omega>\omega_{s} .
\end{array}
$$

This means that up to frequency $\omega_{s}$ the pb is more ordered with respect to $\mathrm{db}$. Over this frequency the contrary occurs. It is to note (see Figs. 1 and 8) that at the frequency $\omega_{s}$ the

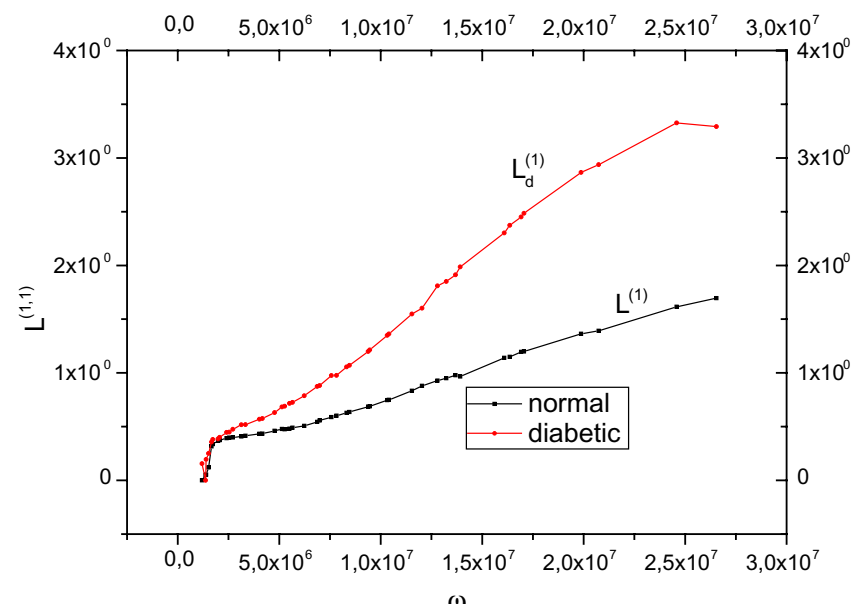

Fig. 7. Displacement conductibility coefficient for $\mathrm{pb}$ and $\mathrm{db}$ (Eq. (24)). 


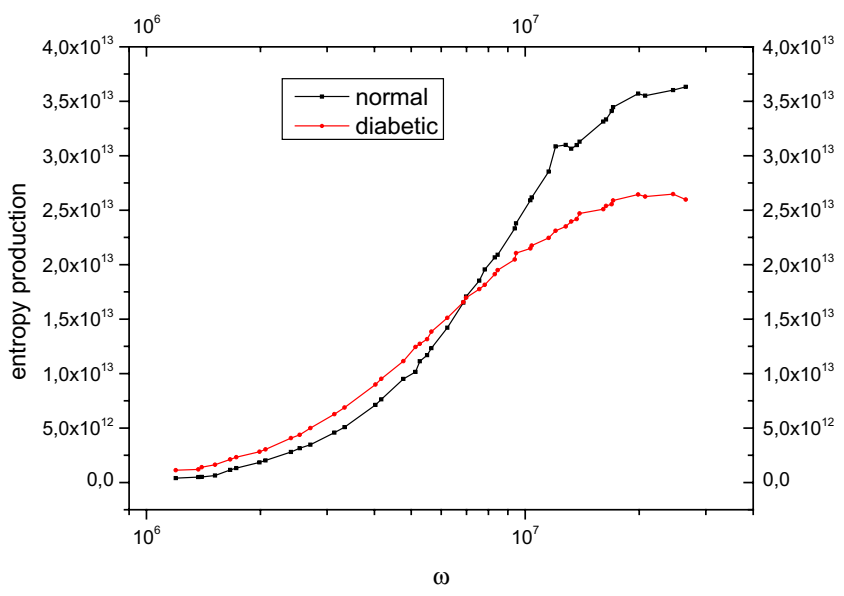

Fig. 8. Trend of entropy production for pb and $\mathrm{db}$ (Eq. (45)).

function $P^{(0)}$ has a minimum (for both $\mathrm{db}$ and $\mathrm{pb}$ ) and $P^{(1)}$ has a maximum (for both $\mathrm{db}$ and $\mathrm{pb}$ ). At present, we are not able to explain this by a biological point of view, but it may be that this point will change with the variations of level of glucose.

\section{References}

${ }^{1}$ H. A. Neil, W. Gatling, H. M. Mather, A. V. Thompson, M. Thorogood, G. H. Fowler, R. D. Hill and J. I. Mann, The Oxford Community Diabetes Study: Evidence for an increase in the prevalence of known diabetes in Great Britain, Diab. Med. 4, 539 (1987).

${ }^{2} \mathrm{P}$. Jeppesen and T. Bek, The occurrence and causes of registered blindness in diabetes patients in Arhus County, Denmark, Acta Ophthalmol. Scand. 82(5), 526 (2004).

${ }^{3}$ R. Huxley, F. Barzi and M. Woodward, Excess risk of fatal coronary heart disease associated with diabetes in men and women: Meta-analysis of 37 prospective cohort studies, BMJ 332, 73 (2006).

${ }^{4}$ G. Remuzzi, M. Macia and P. Ruggenenti, Prevention and treatment of diabetic renal disease in Type 2 Diabetes: The BENEDICT Study, J. Am. Soc. Nephrol. 17(4), S90 (2006).

${ }^{5}$ S. Abdalla, S. S. Al-ameer and S. H. Al-Magaishi, Electrical properties with relaxation through human blood, Biomicrofluidics 4(3), 034101 (2010).

${ }^{6} \mathrm{~S}$. Abdalla, Low frequency dielectric properties of human blood, IEEE Trans. Nanobiosci. 10(2), 113 (2011).

${ }^{7}$ S. R. De Groot and P. Mazur, Non-Equilibrium Thermodynamics (Dover Publication, New York, 1984).

${ }^{8}$ G. A. Kluitenberg, On dielectric and magnetic relaxation phenomena and non-equilibrium thermodynamics, Physica 68(1), 75 (1973).

${ }^{9}$ G. A. Kluitenberg, On dielectric and magnetic relaxation phenomena and vectorial internal degrees, Physica A 87(2), 302 (1977).
${ }^{10}$ G. A. Kluitenberg, On vectorial internal variables and dielectric and magnetic relaxation phenomena, Physica A 109, 91 (1981).

${ }^{11}$ V. Ciancio, F. Farsaci and G. Di Marco, A method for experimental evaluation of phenomenological coefficients in media with dielectric relaxation, Physica B 387, 130 (2007).

${ }^{12}$ V. Ciancio, F. Farsaci and P. Rogolino, Phenomenological approach on wave propagation in dielectric media with two relaxation times, Physica B 404, 320 (2009).

${ }^{13}$ V. Ciancio, F. Farsaci and P. Rogolino, On a thermodynamical model for dielectric relaxation phenomena, Physica B 405, 174 (2010).

${ }^{14}$ F. Farsaci, V. Ciancio and P. Rogolino, Mechanical model for relaxation phenomena in viscoanelastic media of order one, Physica B 405, 3208 (2010).

${ }^{15}$ F. Farsaci and P. Rogolino, An alternative dielectric model for low and high frequencies: A non-equilibrium thermodynamic approach, J. Non-Equilib. Thermodyn. 37(1), 27 (2012).

${ }^{16}$ H. P. Schwan, Electrical properties of blood and its constitutents: Alternating current spectroscopy, Blut 46(4), 185 (1983).

${ }^{17}$ T. Chelidze, Dielectric spectroscopy of blood, J. Non-Cryst. Solids 305, 285 (2002).

${ }^{18}$ M. S. Olufsen, J. T. Ottesen, H. T. Tran, L. M. Ellwein, L. A. Lipsitz and V. Novak, Blood pressure and blood flow variation during postural change from sitting to standing - modeling and experimental validation, J. Appl. Physiol. 99, 1523 (2005).

${ }^{19}$ N. S. Selim, Comparative study on the effect of radiation on whole blood and isolated red blood cells, Rom. J. Biophys. 20(2), 127 (2010).

${ }^{20}$ M. C. De Rosa, M. T. Sanna, I. Messana, M. Castagnola, A. Galtieri, E. Tellone, R. Scatena, B. Botta, M. Botta and B. Giardina, Glycated human hemoglobin HbAlc: Functional characteristics and molecular modeling studies, Biophys. Chem. 72, 323 (1998).

${ }^{21}$ J. L. Wautiera and P. J. Guillausseaub, Diabetes, advanced glycation endproducts and vascular disease, Vasc. Med. 3, 131 (1998).

${ }^{22} \mathrm{~S}$. Abdalla, Complex permittivity of blood cells and E. coli suspensions, J. Mol. Liq. 160(3), 130 (2011).

${ }^{23}$ F. Farsaci, E. Tellone, M. Cavallaro, A. Russo and S. Ficarra, Low frequency dielectric characteristics of human blood: A non-equilibrium thermodynamic approach, J. Mol. Liq. 188, 113 (2013).

${ }^{24}$ N. G. McCrum, B. E. Read and G. Williams, An Elastic and Dielectric Effects in Polymeric Solids (John Wiley and Sons Ltd., London, 1967).

${ }^{25}$ A. Lapolla, L. Molin and P. Traldi, Protein glycation in diabetes as determined by mass Spectrometry, Int. J. Endocr. 11, 2013 (2013).

${ }^{26}$ L. Ang, V. Palakodeti, A. Khalid, S. Tsimikas, Z. Idrees, P. Tran, P. Clopton, N. Zafar, G. Bromberg-Marin, S. Keramati and E. Mahmud, Elevated plasma fibrinogen and Diabetes Mellitus are associated with lower inhibition of platelet reactivity with clopidogrel, J. Am. Coll. Cardiol. 52(13), 1052 (2008). 